\title{
Divergence between bread wheat and Triticum militinae in the powdery mildew resistance QPm.tut- $4 A$ locus and its implications for cloning of the resistance gene
}

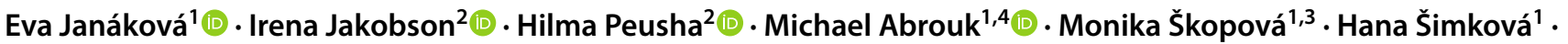

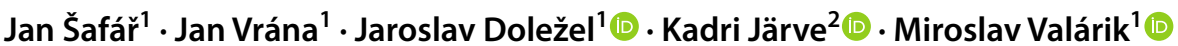

Received: 10 August 2018 / Accepted: 3 December 2018 / Published online: 7 December 2018

(c) The Author(s) 2018

\begin{abstract}
A segment of Triticum militinae chromosome $7 \mathrm{G}$ harbors a gene(s) conferring powdery mildew resistance which is effective at both the seedling and the adult plant stages when transferred into bread wheat (T. aestivum). The introgressed segment replaces a piece of wheat chromosome arm $4 \mathrm{AL}$. An analysis of segregating materials generated to positionally clone the gene highlighted that in a plant heterozygous for the introgression segment, only limited recombination occurs between the introgressed region and bread wheat 4A. Nevertheless, 75 genetic markers were successfully placed within the region, thereby confining the gene to a $0.012 \mathrm{cM}$ window along the $4 \mathrm{AL}$ arm. In a background lacking the $P h l$ locus, the localized rate of recombination was raised 33 -fold, enabling the reduction in the length of the region containing the resistance gene to a $480 \mathrm{kbp}$ stretch harboring 12 predicted genes. The substituted segment in the reference sequence of bread wheat $\mathrm{cv}$. Chinese Spring is longer (640 kbp) and harbors 16 genes. A comparison of the segments' sequences revealed a high degree of divergence with respect to both their gene content and nucleotide sequence. Of the 12 T. militinae genes, only four have a homolog in cv. Chinese Spring. Possible candidate genes for the resistance have been identified based on function predicted from their sequence.
\end{abstract}

\section{Introduction}

Communicated by Hermann Buerstmayr.

Electronic supplementary material The online version of this article (https://doi.org/10.1007/s00122-018-3259-3) contains supplementary material, which is available to authorized users.

Miroslav Valárik

valarik@ueb.cas.cz

1 Institute of Experimental Botany of the Czech Academy of Sciences, Centre of the Region Haná for Biotechnological and Agricultural Research, Šlechtitelů 31, 78371 Olomouc, Czech Republic

2 Department of Chemistry and Biotechnology, Tallinn University of Technology, Akadeemia tee 15, 19086 Tallinn, Estonia

3 Present Address: Limagrain Central Europe Cereals, s.r.o., Hrubčice 111, 79821 Bedihošt', Czech Republic

4 Present Address: Biological and Environmental Science and Engineering Division, King Abdullah University of Science and Technology, Thuwal 23955-6900, Kingdom of Saudi Arabia
Disease imposes an important constraint on crop productivity and is most effectively and sustainably managed by breeding cultivars harboring genetic resistance. The ability of many pathogens to overcome host resistance means that the discovery of additional sources of resistance is a must for any crop improvement program. Since the gene pool of bread wheat (Triticum aestivum) has been so extensively narrowed by more than a century of intensive breeding (Feuillet et al. 2008), the search for novel resistance genes needs to be extended to older materials such as landraces and even to related cultivated and wild species (Mondal et al. 2016; Zamir 2001). Over many years, wheat cytogeneticists and breeders have succeeded in developing a diverse collection of germplasm harboring introgression segments of variable length, many of which have targeted the introduction of genes conditioning resistance to leaf pathogens (King et al. 2017; Valkoun 2001). Advances in genomic technologies have enabled a much greater precision than has been possible hitherto in the characterization of these materials (Abrouk et al. 2017; Tiwari et al. 2014; Winfield et al. 2016). 
A number of introgressed genes conferring resistance to a fungal pathogen have been successfully isolated in hexaploid wheat: these currently comprise two conferring resistance to Puccinia triticina (Huang et al. 2003; Thind et al. 2017), five to $P$. graminis (Mago et al. 2015; Periyannan et al. 2013; Saintenac et al. 2013; Steuernagel et al. 2016) and one to Blumeria graminis (Hurni et al. 2013). B. graminis (commonly referred to as powdery mildew) epiphytotics can induce significant yield losses (Conner et al. 2003; Leath and Bowen 1989). A number of major resistance genes (Pm genes) have been identified (www.wheat.pw.usda.gov/cgibin/GG3/browse.cgi?class=gene); as most of these confer race-specific resistance, they are prone to being overcome by the rapidly evolving pathogen. Often durable form of resistance, referred to as adult plant resistance (APR), is typically conditioned by multiple genes, each conferring a small, but cumulative effect. As a result, uncovering its genetic basis normally requires quantitative trait locus (QTL) analysis. A meta-analysis (Lillemo and Lu 2015) has revealed that such genes are dispersed over 24 QTL-harboring regions, located on 18 of the 21 wheat chromosomes.

The tetraploid bread wheat relative T. militinae (genome formula $A^{t} G$ ) is generally considered to be a spontaneous mutant of T. timopheevii (Dorofeyev et al. 1976), although it has also been suggested to have arisen from an introgressive hybridization between T. timopheevii and the $\mathrm{BA}^{\mathrm{u}}$ tetraploid T. carthlicum (Järve et al. 2002). Derivatives of a wide cross between the bread wheat cultivar (cv.) Tähti (genome formula $\mathrm{BA}^{\mathrm{u}} \mathrm{D}$ ) and T. militinae include a selection (line 8.1) which harbors $T$. militinae segments incorporated within chromosomes 1A, 1B, 2A, 4A, 5A, 5B and 7A (Jakobson et al. 2006). Among other traits introduced from T. militinae, line 8.1 exhibits a marked improvement in the level of powdery mildew resistance expressed at both the seedling and adult plant stages. According to a QTL analysis, the genetic basis of this resistance is dominated (respectively, $33 \%$ and $54 \%$ of the variance shown by seedlings and adult plants) by a genes within a segment located near the distal end of chromosome arm 4AL, referred to as QPm.tut-4A (Jakobson et al. 2006). When present in a cv. Tähti background, the resistance decreases the number of secondary haustoria formed by the pathogen and enhances host cell apoptosis (Islamov et al. 2015). The gene (or genes) present within $Q P$ m.tut-4A, which acts in a race non-specific manner (Jakobson et al. 2012), is located in T. militinae itself on chromosome 7G (Abrouk et al. 2017). In a hybrid between wild-type bread wheat and the introgression line, little recombination occurs between the T. militinae segment and the segment of chromosome 4AL which has been replaced in line 8.1. However, it has been possible to define the genetic length and position of the segment to a $2.5 \mathrm{cM}$ interval (Jakobson et al. 2012). Here, the focus was to isolate the gene(s) which determine the QPm.tut-4A resistance. By deploying a range of genetic and genomic strategies, it has proved possible to identify a small number of candidate genes and to characterize and contrast the sequences which originated from chromosome $7 \mathrm{G}$ with those which they replaced on $4 \mathrm{~A}$.

\section{Materials and methods}

\section{Plant materials and mapping populations}

The Jakobson et al. (2012) mapping population comprised $98 \mathrm{~F}_{2}$ progeny bred from the cross cv. Chinese Spring (CS) $\times$ line 8.1 ; this was extended for the purpose of increasing the level of resolution by self-pollinating $\mathrm{F}_{2}$ individuals which were heterozygous for QPm.tut-4A through to the $\mathrm{F}_{5}$. In addition, a second mapping population (hereafter referred to as the "phl population") was created by crossing the CS phlb mutant (Sears 1977) with T312.30.38.16, a derivative of introgressive line 8.1 in which the only $T$. militinae segment present was the one harboring QPm.tut-4A. In order to derive a parental plant which was both homozygous for the $p h l b$ allele and heterozygous for the segment harboring QPm.tut-4A, the resulting $\mathrm{F}_{1}$ hybrid was back-crossed to the phlb mutant. Marker-assisted selected $\mathrm{BC}_{1} \mathrm{~F}_{1}$ individuals were then allowed to self-pollinate. Selection for the $p h l b$ allele was performed using a multiplex PCR assay involving the markers AWJL3, PSR128, PSR2120 and PSR574 (Roberts et al. 1999), while the QPm.tut-4A harboring segment was marked by $o w m 76$ and owm 96 . The subsequent generations $\left(\mathrm{BC}_{1} \mathrm{~F}_{3}\right.$ and further) were not employed for this study due to their low viability and fertility presumably caused by extensive chromosomal rearrangements associated with the action of phl. Doubled haploid line DH397 containing the T. militinae resistance locus only on $4 \mathrm{AL}$ was derived from cross Tähti $\times 8.1$ (Jakobson et al. 2012). A bacterial artificial chromosome (BAC) library was constructed from the DNA of 4AL telosomic chromosome flow sorted from 4AL ditelosomic line carrying the 4A T. militinae introgression (Jakobson et al. 2012). Grain of the CS aneuploid stocks nullisomic 4A-tetrasomic 4B and nullisomic 4A-tetrasomic $4 \mathrm{D}$, required to validate the chromosome specificity of newly developed markers, was provided by the National BioResource Centre (Kyoto, Japan). A doubled haploid line (DH81) carrying the same T. militinae translocations which determined the powdery mildew resistance as line 8.1 (Jakobson et al. 2012) served as a further control. Finally, the bread wheat cv. Kanzler was used a susceptible host in experiments involving powdery mildew inoculations. 


\section{Phenotyping for powdery mildew resistance}

Seedling resistance to powdery mildew was scored using an assay based on detached first seedling leaves of 10-dayold plants. Each leaf was cut into four segments, each of which was then laid in a Petri dish containing $0.6 \%$ agar supplemented with $0.35 \% \mathrm{w} / \mathrm{v}$ benzimidazol. The leaf segments were inoculated with four different isolates $(2.1,9.8$, 13 and 14), as described by Jakobson et al. (2012). The Petri dishes were held at $17.5^{\circ} \mathrm{C}$, and the response was evaluated after 10 days using the $0-9$ scale devised by Lutz et al. (1992). For each self-pollinated recombinant line selected from $\mathrm{F}_{2}$-derived $\mathrm{F}_{3-5}$ families, up to 30 progeny homozygous in the QPm.tut-4A region were phenotyped. The resistance status of each recombinant line was verified by comparing its progeny scores with scores of 12-16 progeny of homozygous nonrecombinant sister line selected from the same self-pollination.

\section{The development of markers used for high-density genetic mapping}

The informativeness of a potential marker was first assessed via an in silico inspection of the chromosomespecific survey sequences of 4AL-7G (Abrouk et al. 2017) and the chromosome survey sequences (CSS) of $4 \mathrm{AL}^{\mathrm{CS}}$ generated by IWGSC (2014). The choice of sequences was governed by the need to saturate the genetic map of the QPm.tut-4A region, so it was based on a virtual gene order of the chromosome 4A represented by GenomeZippers (Abrouk et al. 2017, Hernandez et al. 2012). 4AL CSS scaffolds obtained from the GenomeZipper delimited by the markers flanking the QPm.tut- $4 A$ segment were aligned with $4 \mathrm{AL}-7 \mathrm{G}$ sequence scaffolds using the BlastN algorithm (Altschul et al. 1990). Only low-copy sequences associated with a nucleotide identity of $>95 \%$ were considered for marker development, and those containing short indels were preferred. Where no indel was identifiable, markers were based on single-nucleotide polymorphisms using the cleaved amplified polymorphic sequence approach (Michaels and Amasino 1999). PCR primers were designed using Primer3 software (Untergasser et al. 2012). To ensure specificity for $4 \mathrm{AL}$, given the presence of homoeologous sequence on 7AS and 7DS, primers were positioned to ensure the presence of a variant nucleotide close to $3^{\prime}$ end in the homoeologous sequences. Finally, the specificity of the putative amplicons was verified by a BlastN search against the whole wheat genome CSS (IWGSC 2014). A second marker discovery strategy profited from a BAC library-based, established CS 4AL-specific physical map (IWGSC 2018, URGI; urgi. versailles.inra.fr/). BAC clones making up the set of contigs which covered the QPm.tut- $4 A$ region were selected from the minimal tiling path and sequenced. Subsequently, sequence scaffolds positioned at the target location were employed for marker development based on the first strategy. A final strategy designed to extend the saturated portion of the QPm.tut-4A region beyond what was achievable using the first two strategies was based on the sequence of the $T$. dicoccoides 7AS region (Avni et al. 2017) and on the CS reference sequence WGA v0.4 (www.wheatgenom e.org); this also was informative for identifying additional $4 \mathrm{AL}^{\mathrm{CS}}$ scaffolds for targeted marker development. All primer pairs were tested on a template of CS, DH81, a CS/line 8.1 derivative heterozygous for $Q P$ m.tut- $4 A$, nullisomic 4A-tetrasomic 4B and nullisomic 4A-tetrasomic $4 \mathrm{D}$ and DNA from flow-sorted chromosome arms $4 \mathrm{AL}^{\mathrm{CS}}$ and 4AL-7G amplified according to Šimková et al. (2008). The methods used for PCR amplification and electrophoretic separation are given in the following section, and the primer sequences and associated information are summarized in Table S1. Where the physical position of a marker was uncertain, it was determined by screening 62 threedimensional pools prepared from the minimal tiling path of the CS 4AL-specific physical map.

\section{Genetic mapping}

DNA was extracted using Agencourt ${ }^{\circledR}$ Genfind $^{\circledR}$ v2 magnetic beads (Beckman Coulter Life Sciences, Indianapolis, IN, USA) on a Beckman Coulter ${ }^{\circledR}$ Biomek $^{\circledR} \mathrm{NX}^{\mathrm{P}}$ workstation, as described by Ivaničová et al. (2016). Markers owm82 and Xgwm160 (Röder et al. 1998) were used to select lines in which a recombination event had occurred in the region of the introgression segment in the mapping populations. The full set of markers was applied to genotype those arising from the $\mathrm{CS} \times$ line 8.1 population, while a subset of 19 selected markers was applied to those arising from the phl population. Each $15 \mu \mathrm{L}$ PCR contained $0.01 \%(w / v)$ o-cresolsulphonephtalein, $1.5 \%(w / v)$ sucrose, $0.2 \mathrm{mM}$ of each dNTP, 0.6 U Taq DNA polymerase, $1 \mu \mathrm{M}$ of each primer, $10 \mathrm{mM}$ Tris- $\mathrm{HCl}, 50 \mathrm{mM} \mathrm{KCl}$, $1.5 \mathrm{mM} \mathrm{MgCl}_{2}$ and $0.1 \%(v / v)$ Triton X-100. The template comprised either 10-20 ng genomic DNA or 5 ng DNA amplified from $4 \mathrm{AL}^{\mathrm{CS}}$ or $4 \mathrm{AL}-7 \mathrm{G}$. The reaction conditions consisted of an initial denaturation step of $95^{\circ} \mathrm{C} / 5 \mathrm{~min}$, followed by 40 cycles of $95^{\circ} \mathrm{C} / 30 \mathrm{~s}$, an optimized annealing temperature (Table S1) for $30 \mathrm{~s}$ and $72{ }^{\circ} \mathrm{C}$ for $30 \mathrm{~s}$ per $500 \mathrm{bp}$ amplicon length; the reactions were completed with an elongation step of $72{ }^{\circ} \mathrm{C} / 5 \mathrm{~min}$. Cleaved amplified polymorphic sequence assays were completed with a digestion using the appropriate restriction endonuclease. The amplicons were electrophoretically separated through $4 \%$ non-denaturing polyacrylamide gels and visualized by ethidium bromide staining. 


\section{Construction of the physical map}

Sequence scaffolds taken from IWGSC RefSeq v1.0 (IWGSC 2018) were used to span the genomic region of CS 4AL replaced by the QPm.tut-4A segment in line 8.1. To obtain the corresponding sequence from T. militinae, a chromosome walking approach was initiated. A chromosome-specific BAC library designated TaaPmt4ALhA (www.olomouc.ueb.cas.cz/dna-libraries/cereals) was constructed from flow-sorted $4 \mathrm{AL}-7 \mathrm{G}$ chromosome arms according to the Šimková et al. (2011) protocol. BAC library plate pools were generated by mixing $40 \mu \mathrm{L}$ of the bacterial culture from each well of a given plate, centrifuging ( $10 \mathrm{~min}$ at $2700 \mathrm{~g}$ ), suspending the precipitate in $0.5 \mathrm{~mL} \mathrm{TE}$ and boiling for $30 \mathrm{~min}$. The suspension was then re-centrifuged $(60 \mathrm{~min}$ at $2700 \mathrm{~g}$ ), and a $450-\mu \mathrm{L}$ aliquot of the supernatant was diluted $100-$ fold. The subsequent PCRs used as template a $1.5-\mu \mathrm{L}$ aliquot of the diluted plate pool DNA. The plate pools were screened with the markers owm 169 and owm 228 , together with other codominant markers located within the CS 4AL segment replaced by QPm.tut-4A (owm156, owm136, owm221, owm209, owm227, owm236, owm139 and $o w m 235$ ). Row and column pools of positive plates were prepared and used in the same way as plate pools. Selected BAC clones were sequenced on a MiSeq instrument (Illumina Inc., San Diego, CA, USA) using a Nextera DNA Library Prep Kit (Illumina) according to the manufacturer's protocol. Paired-end reads were assembled by Ray software (Boisvert et al. 2010). Insertion site-based polymorphism or site-specific presence/absence variation markers were developed from the ends of sequenced BAC clones and used for a further round of BAC library screening. The procedure was iterated until the physical map had been assembled.

\section{The identification and sequence analysis of candidate genes}

The gene content along the QPm.tut-4A segment on 4AL$7 \mathrm{G}$ was annotated using the TriAnnot pipeline (Leroy et al. 2011, www6.inra.fr/decodage/TriAnnot). Predicted genes were subjected to a BlastP search against the set of non-redundant protein sequences (www.ncbi.nlm.nih.gov/ BLAST), and conserved domains of putative proteins were annotated using the NCBI Conserved Domain Database (Marchler-Bauer et al. 2017) and the MOTIF search tool (www.genome.jp/tools/motif/) based on the Pfam database (Finn et al. 2016). Searches for homologs and the comparison of candidate gene sequences were based on the BlastN algorithm, applying a threshold of $90 \%$ identity and $60 \%$ coverage.

\section{Reverse transcription PCR}

Segments of the first leaf of 10-day-old seedlings of the doubled haploid line DH397 and line T312.30.38.16 were collected 0 -, 24- and 48-h post-inoculation with powdery mildew and snap frozen in liquid nitrogen. Leaf segments from three independent inoculations were bulked for the purpose of RNA extraction, and negative control samples were formulated from non-inoculated leaf segments. The leaf tissue was homogenized using ball mill MM 301 (Retsch, Haan, Germany) with three $3 \mathrm{~mm}$ tungsten beads at $30 \mathrm{~Hz}$ for $45 \mathrm{~s}$, and total RNA was extracted using a miRNeasy Mini Kit (Qiagen Inc., Hilden, Germany) according to the manufacturer's protocol. The synthesis of the first cDNA strand was achieved using a Transcriptor High Fidelity cDNA Synthesis Kit (Roche Life Sciences, Indianapolis, IN, USA) based on an anchored-oligo(dT) ${ }_{18}$ primer. Gene-specific primers were designed for all 12 candidate genes such that the amplicons derived from gDNA differed in length from those derived from cDNA (Table S2). A portion of the Actin gene (GenBank accession number AB181991.1) was used as the reference sequence. The procedures used for PCR and electrophoretic separation were as described above.

\section{Results}

\section{High-resolution genetic mapping around QPm. tut-4A}

A set of 102 new genetic markers was developed to saturate the genetic map in the region of QPm.tut-4A (Figs. 1, 2, Table S1). Majority of these were developed using GenomeZipper and 4AL-specific BAC clones. Besides markers owm 16 and owm39, polymorphism was inferred using the $4 \mathrm{AL}-7 \mathrm{G}$ and $4 \mathrm{AL}^{\mathrm{CS}}$ survey sequences (Abrouk et al. 2017, IWGSC 2014). Segregation patterns observed in the extended CS $\times 8.1$ mapping population were used to establish marker order. The genotyping of 8425 individuals with respect to owm 82 and $X g w m 160$ revealed 30 new recombination events. Testing these recombinant individuals for their reaction to the four powdery mildew isolates showed that the resistance phenotype was fully correlated with the presence of the T. militinae segment-the disease scores for the four isolates were, respectively, 0.5, 0.4, 0.1, 1.1 in the presence of the segment, and 3.3, 2.5, 2.6, 3.8 in its absence. The net effect of the mapping was reducing the genetic length of the segment harboring QPm.tut-4A to $0.012 \mathrm{cM}$ (Fig. 1). The phl population was derived from the self-pollination of 22 (out of 107 screened) $\mathrm{BC}_{1} \mathrm{~F}_{1}$ ([line $8.1 \times p h 1 b] \times p h 1 b)$ selections which were simultaneously homozygous for the $p h 1 b$ allele and heterozygous for the QPm.tut-4A segment. The genotyping of the resulting 1255 


\section{A CS $\times 8.1 \mathrm{Ph1}$}
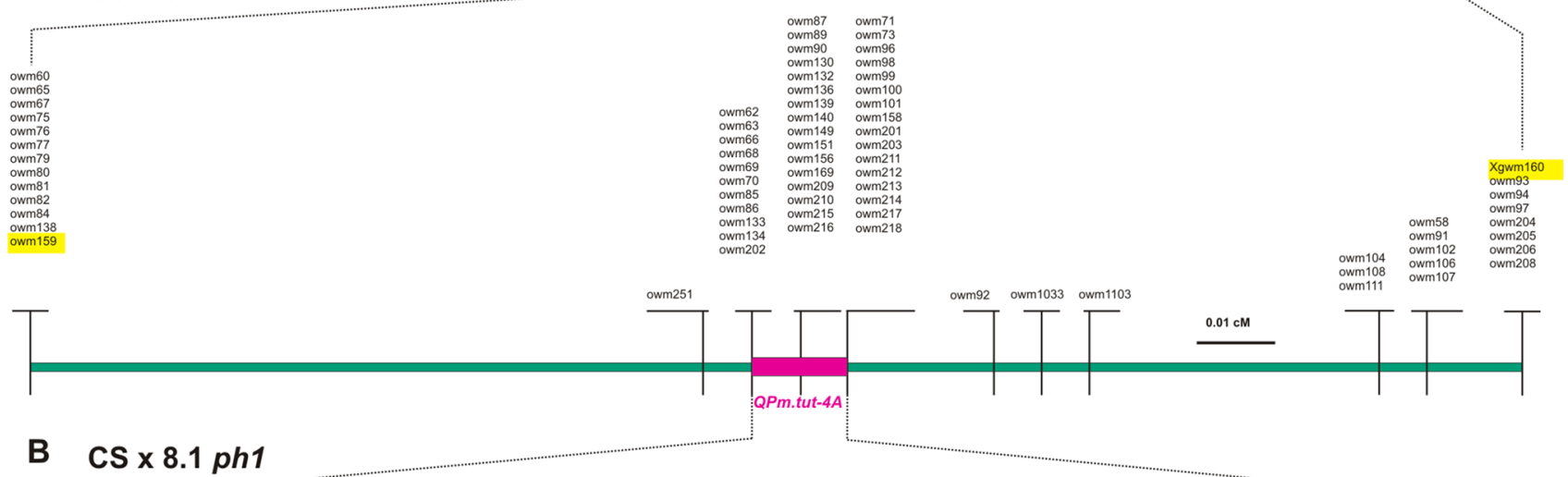

\section{B CS $\times 8.1 p h 1$}

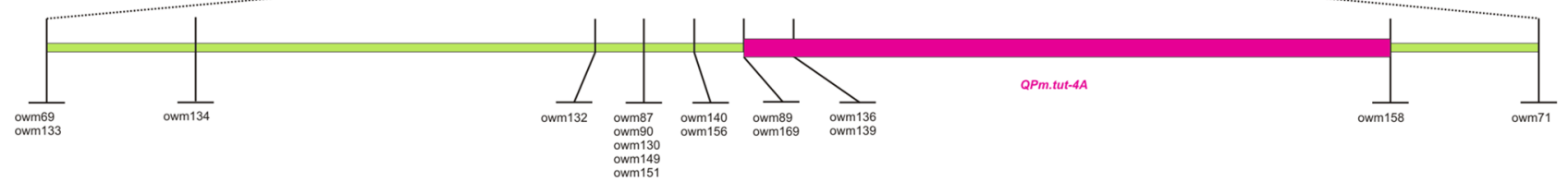

Fig. 1 A high-density genetic map of the region harboring QPm.tut$4 A$. a Marker segregation displayed by 8519 progeny bred from the $\mathrm{CS} \times 8.1$ mapping population (including the $\mathrm{F}_{2}$ generation described at Jakobson et al. 2012) defines the genetic length of the QPm.tut-4A segment (marked in red) to $0.012 \mathrm{cM}$, lying within a $0.18 \mathrm{cM}$ window within chromosome 4AL. The flanking markers used for identi- fication of recombination events within the segment are highlighted in yellow. b The level of mapping resolution achieved was enhanced through the use of the phl mapping population, which provided an additional 30 new recombination events, thereby splitting the $Q P m$. tut- $4 A$ region into eight subregions

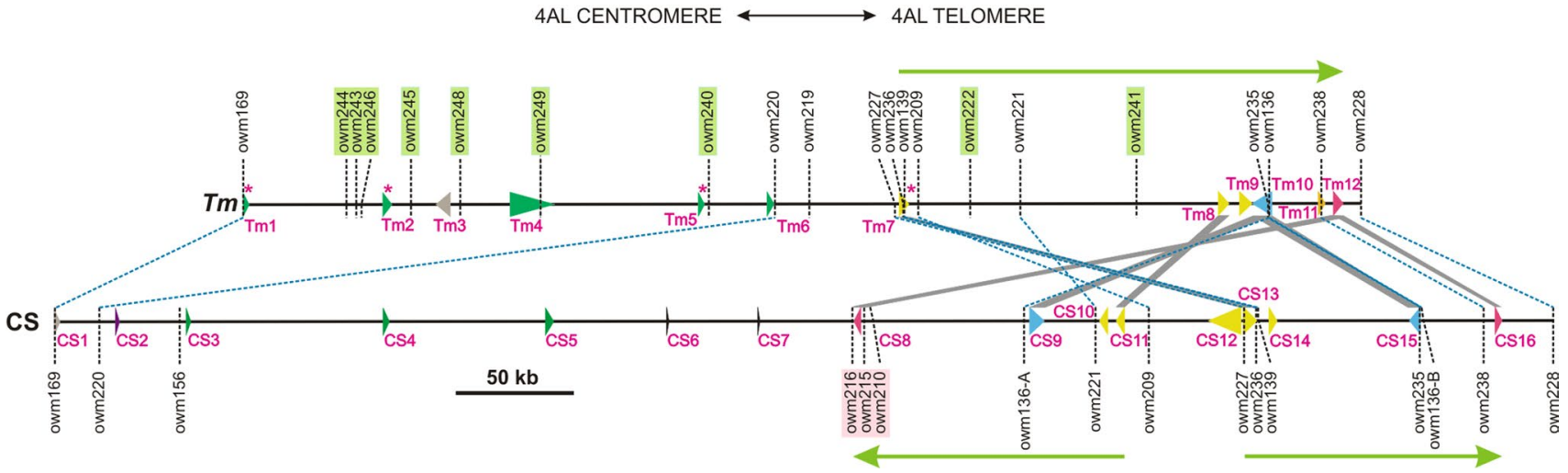

Fig. 2 Physical maps showing the gene content of the $480.2 \mathrm{kbp}$ QPm.tut-4A segment inherited from T. militinae and the $640.8 \mathrm{kbp}$ segment of chromosome 4AL replaced by the introgression in line 8.1. The predicted genes (Tm1-Tm12 and CS1-CS16) are depicted by arrowheads indicating their orientation. The size of the arrowheads is proportional to the length of the gene, and their color reports the presence of conserved domains: green: CC-(LRR)-NB-ARC; yellow: PGG; orange: ANK and PGG; blue: LRR/MAL/PK; red: patatin-like; black: PMEI-like; and gray: uncharacterized protein. $T m$ and $C S$ genes linked by gray lines share sequence identity in their coding region of at least $90 \%$ at a coverage of $\geq 60 \%$ (a coverage $<80 \%$ applied only to the putative pseudogene $C S 15$ ). Tm genes which produced a detectable transcript are indicated by a red asterisk. Shared markers are linked by a blue dashed line, those marking T. militinae but not CS genomic sequence, or vice versa, are highlighted in, respectively, light green and light red. The T. militinae region bounded by owm 227 and owm 228 was present in CS as two tandemly arranged, tail-to-tail orientated copies, as indicated by the green arrows 
$\mathrm{BC}_{1} \mathrm{~F}_{2}$ progeny revealed 155 recombination events between owm82 and $\mathrm{Xgwm} 160$, equivalent to a 33 -fold increase in recombination as compared to the rate observed in the presence of Phl. The $0.012 \mathrm{cM} \mathrm{QPm.tut-4A}$ region was thereby split into eight subregions (Fig. 1b).

\section{The physical map of the QPm.tut-4A segment}

Six additional markers for the QPm.tut-4A region were developed by inspection of the three CS reference sequence IWGSC WGA v0.4 scaffolds (134864, 108602 and 47761) (www.wheatgenome.org) selected on the basis of markers lying within the owm169-owm158 interval (Table S1). The owm 228 marker was derived from same scaffold (134864) which contained the QPm.tut-4A flanking marker owm 169. The application of these markers to the set of recombinant segregants defined the physical length of the CS sequence replaced by the $Q P m . t u t-4 A$ introgression to $640.8 \mathrm{kbp}$ (Fig. 2). A 4AL-7G-specific BAC library was constructed in an effort to acquire sequences in the $Q P m$. tut $-4 A$ region of line 8.1 ; the mean insert size harbored by the 43,008 clones generated provided a 6.8 -fold coverage of the 4AL-7G chromosome arm (www.olomouc.ueb.cas. cz/dnalib/taapmt4alha). Pools of the set of 112 plates were assembled to perform a PCR screen based on the flanking markers owm169 and owm 228, along with eight codominant markers (owm156, owm136, owm221, owm209, owm227, owm236, owm 139 and owm235) mapping within the region (Fig. 2). One to seven positive BAC clones per marker were sequenced so that their end sequences could be used for the next round of chromosome walking-based marker development: 17 new markers were designed in this way (Table S1). In total, $26 \mathrm{BAC}$ clones were sequenced to provide a complete sequence of the QPm.tut-4A region in line 8.1: The physical length of the segment bounded by owm 169 and owm 228 was $480.2 \mathrm{kbp}$.

\section{Annotation and comparative analysis of the QPm. tut-4A segment}

The T. militinae region was $25 \%$ shorter than the CS one (480.2 vs. $640.8 \mathrm{kbp}$ ). Gene annotation suggested that the QPm.tut-4A segment harbored 12 high-confidence (HC) protein-encoding genes (denominated Tm1-Tm12, Fig. 2, Table 1), while the $4 \mathrm{AL}$ region of CS harbored $16 \mathrm{HC}$ genes (CS1-CS16, Table 2). The intergenic regions displayed no sequence similarity. A comparison of the T. militinae and CS genes based on a threshold of $90 \%$ identity and $60 \%$ coverage implied that only four of the T. militinae genes ( $T m 7, T m 8, T m 10$ and $T m 12)$ shared an appreciable level of homology with members of the CS set (CS8, CS9, CS11, CS13, CS15 and CS16) (Table 1, Fig. 2). The sequence of

Table 1 Predicted candidate genes present in the QPm.tut-4A segment introgressed from T. militinae into line 8.1

\begin{tabular}{|c|c|c|c|c|c|c|c|}
\hline No. & $\begin{array}{l}\text { Putative conserved protein } \\
\text { domains }\end{array}$ & No. of exons & $\begin{array}{l}\text { Length of pro- } \\
\text { tein sequence } \\
\text { (aa) }\end{array}$ & $\begin{array}{l}\text { CS homologous genes in the } \\
\text { QPm.tut- } 4 \text { A region }{ }^{\mathrm{a}}\end{array}$ & Identity $(\%)$ & Coverage $(\%)$ & $\begin{array}{l}\text { RNA } \\
\text { expression }^{b}\end{array}$ \\
\hline $\operatorname{Tm} 1$ & $\mathrm{CC} ; \mathrm{NB}-\mathrm{ARC}$ & 2 & 225 & - & - & - & + \\
\hline $\operatorname{Tm} 2$ & CC; LRR; NB-ARC & 2 & 753 & - & - & - & + \\
\hline $\operatorname{Tm} 3$ & - & 3 & 253 & - & - & - & - \\
\hline $\operatorname{Tm} 4$ & CC; LRR; NB-ARC & 7 & 894 & - & - & - & - \\
\hline $\operatorname{Tm} 5$ & CC; LRR; NB-ARC & 2 & 557 & - & - & - & + \\
\hline $\operatorname{Tm} 6$ & CC; LRR; NB-ARC & 2 & 1017 & - & - & - & - \\
\hline $\operatorname{Tm} 7$ & $5 \times$ PGG & 2 & 963 & $\begin{array}{l}\text { TraesCS4A01G450100.2 } \\
\quad(\mathrm{CS} 13)\end{array}$ & 97 & 99 & + \\
\hline $\operatorname{Tm} 8$ & $5 \times \mathrm{PGG}$ & 3 & 764 & $\begin{array}{l}\text { TraesCS4A01G449900 } \\
\quad(\mathrm{CS} 11)\end{array}$ & 90 & 98 & - \\
\hline $\operatorname{Tm} 9$ & $4 \times$ PGG & 2 & 849 & - & - & - & - \\
\hline $\operatorname{Tm} 10$ & LRR; Malectin; Pkinase & 23 & 913 & $\begin{array}{l}\text { TraesCS4A01G449700 (CS9) } \\
\text { TraesCS4A01G450300 } \\
\text { (CS15) }\end{array}$ & $\begin{array}{l}97 \\
98\end{array}$ & $\begin{array}{l}84 \\
60\end{array}$ & - \\
\hline $\operatorname{Tm} 11$ & Ankyrin repeats; PGG & 4 & 636 & - & - & - & - \\
\hline $\operatorname{Tm} 12$ & Patatin-like phospholipase & 5 & 424 & $\begin{array}{l}\text { TraesCS4A01G449600 (CS8) } \\
\text { TraesCS4A01G450400 } \\
\text { (CS16) }\end{array}$ & $\begin{array}{l}98 \\
97\end{array}$ & $\begin{array}{l}100 \\
100\end{array}$ & - \\
\hline
\end{tabular}

$C C$ coiled coil, $N B$-ARC nucleotide-binding adaptor shared by APAF-1, certain $R$ gene products and CED4, $L R R$ leucine-rich repeat, $P G G$ proline-glycine-glycine (domain named for this highly conserved sequence motif found at its start), Pkinase protein kinase

${ }^{a}$ Acronym for each gene used in the body of this manuscript and Fig. 2 is provided in the parentheses

${ }^{\mathrm{b}}$ Detected in first leaves of DH397 and T312.30.38.16 seedlings 
Table 2 Gene content of the segment of CS chromosome arm 4AL replaced by the QPm.tut-4A segment introgressed from T. militinae into line 8.1

\begin{tabular}{|c|c|c|c|c|}
\hline No. & Official designation $^{\mathrm{a}}$ & Putative conserved protein domains & No. of exons & $\begin{array}{l}\text { Length of } \\
\text { protein sequence } \\
\text { (aa) }\end{array}$ \\
\hline CS1 & TraesCS4A01G448900 & $\mathrm{CC}$ & 3 & 313 \\
\hline$C S 2$ & TraesCS4A01G449000 & Transferase & 1 & 472 \\
\hline CS3 & TraesCS4A01G449100 & $\mathrm{CC} ; \mathrm{NB}-\mathrm{ARC}$ & 2 & 353 \\
\hline CS4 & TraesCS4A01G449200 & NB-ARC; LRR & 2 & 838 \\
\hline CS5 & TraesCS4A01G449300 & CC; LRR; NB-ARC & 3 & 1008 \\
\hline CS6 & TraesCS4A01G449400 & Plant invertase/pectin methylesterase inhibitor & 1 & 203 \\
\hline CS7 & TraesCS4A01G449500 & Plant invertase/pectin methylesterase inhibitor & 1 & 206 \\
\hline CS8 & TraesCS4A01G449600 & Patatin-like phospholipase & 5 & 424 \\
\hline CS9 & TraesCS4A01G449700 & LRR; Malectin; Pkinase & 21 & 867 \\
\hline CS10 & TraesCS4A01G449800 & $5 \times$ PGG & 2 & 993 \\
\hline CS11 & TraesCS4A01G449900 & $5 \times \mathrm{PGG}$ & 2 & 1020 \\
\hline CS12 & TraesCS4A01G450000 & $4 \times$ PGG & 3 & 761 \\
\hline CS13 & TraesCS4A01G450100.2 & $5 \times$ PGG & 3 & 984 \\
\hline CS14 & TraesCS4A01G450200 & $5 \times$ PGG & 2 & 1024 \\
\hline CS15 & TraesCS4A01G450300 & LRR; Malectin & 15 & 549 \\
\hline CS16 & TraesCS4A01G450400 & Patatin-like phospholipase & 5 & 424 \\
\hline
\end{tabular}

${ }^{\mathrm{a}}$ The International Wheat Genome Sequencing Consortium RefSeq v1.0 annotation (IWGSC 2018)

the proximal ends of the CS and line 8.1 regions (beyond owm 169) were quite distinct with respect to both their sequence and gene content: The segment from owm 169 to owm 220 was $228 \mathrm{kbp}$ in line 8.1 , but only $19 \mathrm{kbp}$ in CS, and they contained, respectively, six (Tm1-Tm6) and one (CS1) genes. The central part of the segment (owm220-owm 227 in line 8.1 and owm220-owm216 in CS) was longer in CS (322 vs. $51 \mathrm{kbp}$ ) and contained no predicted gene in line 8.1 and CS2-CS7 in CS. The distal region (owm 227-owm 228 in line 8.1 and owm 216-owm 228 in CS) was, respectively, of length $200 \mathrm{kbp}$ (genes Tm7-Tm12) and $299 \mathrm{kbp}$ (CS8-CS16) (Fig. 2). The proximal segment harboring $T m 1-T m 6$ was not represented on CS chromosome 4A. However, four of these genes (Tm1, Tm2, Tm5 and $T m 6$ ) each have a strong homoeolog (Table 3) lying in reverse orientation within a $\sim 410 \mathrm{kbp}$ region of chromosome arm 7AS situated about 17.4 Mbp from the telomere. The position of the QPm.tut-4A region is $41.05 \mathrm{Mbp}$ distant from the $4 \mathrm{AL}$ telomere. The distal part of the region (owm227-owm228) has been duplicated in CS, and the gene content has been differentiated. The two copies are arranged tandemly in a tail-to-tail orientation, resulting in the non-syntenic location of the owm 227 marker (Fig. 2).

\section{Functional characterization of the candidate genes for powdery mildew resistance}

The five genes $T m 1, T m 2$ and $T m 4-T m 6$ each encoded a coiled-coil (CC) domain and a nucleotide-binding (NB) domain, thereby being members of the NB-ARC family (van der Biezen and Jones 1998). Tm2, Tm4 and Tm6 also encode a leucine-rich repeat (LRR) domain, indicating them as members of the disease resistance-associated NLR gene family (Ye and Ting 2008). The shorter length of both Tm1 and Tm5 (Table 1) implies that both are incomplete

Table 3 Homoeologs on CS chromosome arm 7AS of the NLR family genes present in the QPm.tut-4A segment introgressed from T. militinae into line 8.1

\begin{tabular}{lllllll}
\hline No. & CS homoeolog on 7AS & Identity (\%) & Coverage (\%) & $\begin{array}{l}\text { Putative conserved protein } \\
\text { domains of homoeologs }\end{array}$ & $\begin{array}{l}\text { Length of protein } \\
\text { sequence (aa) }\end{array}$ & $\begin{array}{l}\text { Length difference relative to } \\
\text { T. militinae homoeolog (aa) }\end{array}$ \\
\hline Tm1 & TraesCS7A01G038800 & 95 & 100 & CC; NB-ARC & 452 & +227 \\
Tm2 & TraesCS7A01G039300 & 97 & 100 & CC; LRR; NB-ARC & 972 & +219 \\
Tm4 & - & - & - & - & - & - \\
Tm5 & TraesCS7A01G03910 & 98 & 100 & CC; LRR; NB-ARC & 1074 & +517 \\
Tm6 & TraesCS7A01G039400 & 93 & 79 & CC; LRR; NB-ARC & 875 & -142 \\
\hline
\end{tabular}


genes. $T m 10$ is predicted to encode a protein harboring an LRR/malectin/protein kinase (LRR/MAL/PK) domain, also shared by a number of plant disease resistance gene products (Sekhwal et al. 2015); this gene lies in the distal part of the introgression segment and shares homology with both CS9 and the truncated CS15 (Fig. 2). The sequences of the other six $T m$ genes have little or no connection with disease resistance: $\operatorname{Tm} 7-\operatorname{Tm} 9$ each encode multiple PGG domains, $T m 11$ features ankyrin repeats, while $T m 12$ encodes a protein belonging to the patatin-like phospholipase family (Table 1). Four of the $12 \mathrm{Tm}$ genes (Tm1, Tm2, Tm5 and $T m 7)$ produced a detectable level of mRNA, and three of them (Tm1, $T m 2$ and $T m 5$ ) encode NLR proteins: $T m 2$ appears to be the only one of these which is intact.

\section{Discussion}

The introduction into the bread wheat gene pool of genes harbored by species belonging to its secondary and tertiary gene pools represents an attractive strategy for broadening the genetic diversity of a crop which has been intensively bred for over a century. However, the success of the strategy has been not infrequently limited by the simultaneous introgression of linked genes which are deleterious to either productivity and/or product quality. This phenomenon of linkage drag results from the suppression of recombination around an introgressed segment, a phenomenon which also hampers positional cloning, since it magnifies the ratio between physical (in bp) and genetic (in $\mathrm{cM}$ ) distance and prevents efficient high-density mapping. The $T$. militinae segment harboring QPm.tut-4A suffers from exactly this problem. Recent significant progress in the next-generation sequencing technologies allowed development of two new gene cloning approaches called MutRenSeq (Steuernagel et al. 2016) and MutChromSeq (Sánchez-Martín et al. 2016) which can bypass the high-density map construction in gene cloning process. The MutRenSeq approach employing exome capture and sequencing focusses on identification of simultaneous deleterious mutations in single NB-LRRlike gene within mutants with lost resistance. The MutChromSeq uses similar approach consisting in identification of knockout mutations in single gene within mutant lines, but it requires flow sorting of respective chromosomes from all mutant lines and their sequencing. This means that only single dominant major-effect genes can be identified by these approaches. However, the QPm.tut-4A locus is associated with race non-specificity and incomplete resistance which suggests the resistance may be encoded by gene different from major-effect $R$ genes (predominantly NB-LRR-like genes) or by more than one gene (Jakobson et al. 2006, 2012). The MutRenSeq and MutChromSeq approaches are therefore not feasible for the QPm.tut-4A gene/genes cloning. Fortunately, recent advances in DNA technology and the development of sophisticated genomic resources have substantially eased the processes of constructing a high-density map and of acquiring relevant sequence from both the donor and the recipient genomes.

\section{High-density mapping}

According to the Wheat-Composite2004-4A map (wheat. pw.usda.gov/GG3), the markers flanking QPm.tut-4A $(X w m c 232$ and Xgwm160) are separated by $\sim 9 \mathrm{cM}$, which led Jakobson et al. (2012) to suggest that a plant heterozygous for the introgression experiences suppression of recombination in the region, as has been documented for a number of other bread wheat introgression segments (Bariana et al. 2001; Järve et al. 2000; Jia et al. 1996; Lukaszewski 2015). Several genomic resources developed in CS were exploited here to saturate the region with markers in order to fine map the resistance locus, and these greatly enhanced the efficiency with which such markers could be elaborated, especially compared to conventional approaches to marker discovery such as microsatellite screening (Röder et al. 1998) or searching for sequence polymorphism in expressed sequence (e.g. Valárik et al. 2006). The GenomeZipper and chromosome-specific survey sequences (Abrouk et al. 2017, Hernandez et al. 2012, IWGSC 2014) were particularly effective in this context, but use was also made of sequence scaffolds developed in the tetraploid wheat $T$. dicoccoides (Avni et al. 2017) and the BAC contigs defining the chromosome 4AL physical map (IWGSC 2018). Initially, these resources allowed the genetic length of the target to be narrowed to just $0.012 \mathrm{cM}$ (Fig. 1). In a fully homologous situation, such as occurs on wheat chromosome 3B, the mean ratio between $\mathrm{cM}$ and $\mathrm{Mbp}$ in distal regions varies from 0.60 to 0.96 (Choulet et al. 2014). If the recombination between the introgressed segment and the unaltered bread wheat region was unimpeded, the physical length of the segment would have been estimated to be no longer than $20 \mathrm{kbp}$. The fact that the segment's length was measured in hundreds of kbp demonstrated that there was a substantial localized suppression of recombination.

\section{Structural divergence and recombination in the QPm.tut-4A segment}

The pairing of homoeologs in hexaploid wheat is strongly restricted by the action of the Phl locus (Riley and Chapman 1958). The intention of creating the $p h l$ population was to induce a higher rate of recombination between the introgression containing QPm.tut-4A and its presumed homoeologous segment of CS 4AL, since the rate of recombination achieved in the presence of $P h l$ was $<0.4 \%$ (31 out of 8519 ) within the owm82-Xgwm160 
region (Fig. 1): Although this represented a very small genetic distance $(0.18 \mathrm{cM})$, the suppression of pairing/ recombination meant that the physical distance involved was potentially rather large. The effect was enhancing the rate of recombination by 33 -fold. A comparable elevation in the recombination rate induced by removal of the $P h 1$ locus has been reported in a variety of interspecific hybrids (Lukaszewski 1995, Luo et al. 2000). The availability of the genomic sequence of CS (IWGSC 2018) made it possible to identify that the length of the $4 \mathrm{AL}$ segment $(0.012$ cM) replaced by the owm169-owm228 QPm.tut-4A locus in line 8.1 was $640 \mathrm{kbp}$ (Figs. 1,2) and that it harbors 16 predicted genes (Table 2 ). The inferred relationship between genetic and physical distance in the segment was therefore only $0.019 \mathrm{cM}$ per $\mathrm{Mbp}$, which is much lower than the ratio $(0.60-0.96 \mathrm{cM}$ per Mbp) obtained in the distal region of chromosome $3 \mathrm{~B}$ and is even below the ratio associated with the $3 \mathrm{~B}$ centromeric region $(0.05 \mathrm{cM}$ per $\mathrm{Mbp}$ ) in which recombination is known to be repressed (Choulet et al. 2014). This major suppression of recombination reflects the lack of homology between the native wheat and the introgressed QPm.tut-4A locus. The length of the introgressed QPm.tut-4A locus (owm169-owm228) was $480 \mathrm{kbp}$ (Fig. 2), and it harbored 12 predicted genes (Table 1), eight of which lacked a homolog in the CS segment (Table 1, Fig. 2). The CS homologs of the other four genes $(\operatorname{Tm} 7, \operatorname{Tm} 8, \operatorname{Tm} 10$ and $\operatorname{Tm} 12)$ lay in a duplicated segment, and one of the duplicated copies was present in inverted orientation. The region was further disrupted by a number of indels, and all these changes suggest a high level of evolutionary dynamics of the wheat genome. While loss of synteny and sequence divergence are commonplace between homoeologous genomes (Saintenac et al. 2013; Wicker et al. 2003), they can also feature in comparisons made between homologous genomes of different hexaploid wheat cultivars (Mago et al. 2014; Tsõmbalova et al. 2016).

The introgressed QPm.tut-4A locus included three fulllength and two truncated NLR family genes (Table 1). This class of genes is frequently arranged in clusters, which is thought to reflect the outcome of duplication events followed by sequence divergence (Michelmore and Meyers 1998). The coding sequences of the T. militinae NLR-like genes shared only a moderate to a high (73-89\%) level of identity at the nucleotide level. Their putative homoeologs present on chromosome arm 7AS of CS displayed a similar level of sequence relatedness (71-85\%). This chromosomal location confirms the conclusion of Abrouk et al. (2017) that in $T$. militinae itself, the QPm.tut-4A segment is present on chromosome $7 \mathrm{G}$. The $\mathrm{G}$ genome donor is thought to be a member of the Sitopsis section of the genus Aegilops, as is also the donor of the bread wheat B genome (Gornicki et al. 2014). The bread wheat chromosome $4 \mathrm{~A}$ itself is a restructured chromosome composed of a mosaic of segments derived from 4AL, 5AL and 7BS (Devos et al. 1995, Hernandez et al. 2012), and the QPm.tut-4A introgression appears to lie within a part of this region which originated from 7BS, consistent with its transfer following meiotic pairing.

\section{The potential function of the QPm.tut-4A candidates}

The resistance to powdery mildew associated with the presence of the QPm.tut-4A segment was race non-specific and in a cv. Tähti background, accounted for $40 \%$ of the variation in resistance (Jakobson et al. 2012). To date, only few wheat genes associated with APR have been isolated, so unlike the case for race-specific seedling resistance genes, many of which belong to the NLR family, it is not clear what functionality the product of such a gene might have. The Lr34/ Yr18/Pm38 gene, which provides protection against three distinct foliar pathogens, has been shown to encode an ABC transporter (Krattinger et al. 2009), while Lr22a, which confers broad-spectrum APR to leaf rust, encodes an NLR-like protein (Thind et al. 2017), as does the rice NLR family $\mathrm{Pbl}$ gene against panicle blast (Hayashi et al. 2010). Assuming that one of the three NLR family genes $T m 2, T m 4$ and Tm6 represents the most likely candidate for the QPm.tut- $4 A$ resistance, the possible basis of its resistance being race nonspecific needs to be explored. In one scenario, it may be that either two or even all three of the $T m$ genes, which are each individually race-specific, act together to confer apparent race non-specificity. Alternatively, it is possible that one of the three genes is a "defeated" major resistance gene which has retained some residual broad-spectrum effect, as has been demonstrated for some other defeated major resistance genes ( $\mathrm{Li}$ et al. 1999). The latter hypothesis is probably the more plausible, given that the only gene for which a mRNA was detected was $T m 2$, although surprisingly it was possible to detect transcript of the two NLR-like pseudogenes $T m 1$ and $T m 5$. However, the lack of an LRR domain in the Tm2-encoded protein implies that it would be difficult for its product to recognize the pathogen's avirulence signal. An additional candidate is represented by $T m 10$ which encodes a protein containing an LRR, a MAL and a PK domain. A barley protein of this domain composition (HvLEMK1) is known to mediate non-host resistance to powdery mildew, while its wheat ortholog acts to enhance the level of wheat host resistance to powdery mildew (Rajaraman et al. 2016). A comparison of the TaLEMK 1 and the Tm10 protein sequences showed that they share only a $34 \%$ level of identity; this rather low level of homology, combined with the observations that the wheat LEMKI homoeologs map to the group 5 chromosomes and that no $T m 10$ transcript was detected, rules out the possibility that the QPm.tut-4A resistance is conferred by $T m 10$. None of the other $T m$ genes 
encode a product which has been directly associated with disease resistance to date. However, Tm12-which encodes a protein belonging to the patatin-like phospholipase family-remains a candidate since the patatin-like protein AtPLP2 has been shown to represent a component of the cell machinery delivering apoptosis, and therefore makes a contribution toward resistance against an obligate biotroph (La Camera et al. 2009).

Author contribution statement KJ, JD and MV designed the study; MV, EJ, MŠ, HŠ, JŠ and IJ were responsible for marker development, genotyping, BAC library construction, chromosome sorting, data analysis and the construction of the mapping populations. HP performed the phenotypic evaluation and IJ the statistical analysis. The bioinformatics analyses were conducted by EJ and MA, who also contributed to data interpretation. Other experiments were conducted by EJ. The manuscript was drafted by EJ and $\mathrm{MV}$, and all the authors contributed to its editing and proofreading.

Acknowledgements The technical assistance of Marie Seifertová, Radka Tušková, Eva Jahnová and Helena Tvardíková is highly appreciated. This research was financially supported by the Czech Republic Ministry of Education, Youth and Sports (Award LO1204 from the National Program of Sustainability I), by the Czech Science Foundation (Award 18-11688S), the Czech Republic Ministry of Agriculture (Award QK1710302) and by an IUT 193 Grant from the Estonian Ministry of Education and Research. The authors thank the International Wheat Genome Sequencing Consortium (IWGSC) for pre-publication access to IWGSC RefSeq v1.0, the National BioResource Centre (Kyoto, Japan) for providing the CS aneuploid stocks and S. Reader (John Innes Centre, Norwich, UK) for the gift of grain of the phlb mutant. Access to computing and storage facilities owned by parties and projects contributing to the National Grid Infrastructure MetaCentrum provided under the program "Projects of Large Research, Development and Innovation Infrastructures" (CESNET LM2015042) is greatly appreciated.

\section{Compliance with ethical standards}

Conflict of interest On behalf of all authors, the corresponding author confirms that no conflict of interest applies.

Data availability The DNA sequence of the T. militinae-derived $Q P m$. tut- $4 A$ introgression segment has been deposited in GenBank (Accession No. MG672525). Supporting data are available from the corresponding author upon reasonable request.

Funding The study was financially supported by the grants listed in the Acknowledgements section.

Open Access This article is distributed under the terms of the Creative Commons Attribution 4.0 International License (http://creativeco mmons.org/licenses/by/4.0/), which permits unrestricted use, distribution, and reproduction in any medium, provided you give appropriate credit to the original author(s) and the source, provide a link to the Creative Commons license, and indicate if changes were made.

\section{References}

Abrouk M, Balcárková B, Šimková H, Komínková E, Martis M, Jakobson I, Timofejeva L, Rey E, Vrána J, Kilian A, Järve K, Doležel J, Valárik M (2017) The in silico identification and characterization of a bread wheat/Triticum militinae introgression line. Plant Biotechnol J 15:249-256

Altschul SA, Gish W, Miller W, Myers EW, Lipman DJ (1990) Basic local alignment search tool. J Mol Biol 215:403-410

Avni R, Nave M, Barad O, Baruch K, Twardziok SO, Gundlach H, Hale I, Mascher M, Spannagl M, Wiebe K, Jordan KW, Golan G, Deek J, Ben-Zvi B, Ben-Zvi G, Himmelbach A, MacLachlan RP, Sharpe AG, Fritz A, Ben-Davis R, Budak H, Fahima T, Korol A, Faris JD, Hernandez A, Mikel MA, Levy AA, Steffenson B, Maccaferri M, Tuberosa R, Cattivelli L, Faccioli P, Ceriotti A, Kashkush K, Pourkheirandish M, Komatsuda T, Eilam T, Sela H, Sharon A, Ohad N, Chamovitz DA, Mayer KFX, Stein N, Ronen G, Peleg Z, Pozniak CJ, Akhunov ED, Distelfeld A (2017) Wild emmer genome architecture and diversity elucidate wheat evolution and domestication. Science 357:93-97

Bariana HS, Hayden MJ, Ahmed NU, Bell JA, Sharp PJ, McIntosh RA (2001) Mapping of durable adult plant and seedling resistances to stripe rust and stem rust diseases in wheat. Aust J Agric Res 52:1247-1255

Boisvert S, Laviolette F, Corbeil J (2010) Ray: simultaneous assembly of reads from a mix of high-throughput sequencing technologies. J Comput Biol 17:1519-1533

Choulet F, Alberti A, Theil S, Glover N, Barbe V, Daron J, Pingault L, Sourdille P, Couloux A, Paux E, Leroy P, Mangenot S, Guilhot N, Le Gouis J, Balfourier F, Alaux M, Jamilloux V, Poulain J, Durand C, Bellec A, Gaspin C, Safar J, Dolezel J, Rogers J, Vandepoele K, Aury JM, Mayer K, Berges H, Quesneville H, Wincker P, Feuillet C (2014) Structural and functional partitioning of bread wheat chromosome 3B. Science 345:1249721

Conner RL, Kuzyk AD, Su H (2003) Impact of powdery mildew on the yield of soft white spring wheat cultivars. Can J Plant Sci 83:725-728

Devos KM, Dubcovsky J, Dvorak J, Chinoy CN, Gale MD (1995) Structural evolution of wheat chromosomes 4A, 5A, and 7B and its impact on recombination. Theor Appl Genet 91:282-288

Dorofeyev VF, Jakubtsiner MM, Rudenko MI, Migushova EF, Udachin RA, Merezhko AF, Semenova LV, Novikova MV, Gradchaninova OD, Shitova IP (1976) The wheats of the world. In: Brezhnev DD, Dorofeyev VF (eds), Kolos Publication, Leningrad, 487 pp. (in Russian)

Feuillet C, Langridge P, Waugh R (2008) Cereal breeding takes a walk on the wild side. Trends Genet 24:24-32

Finn RD, Coggill P, Eberhardt RY, Eddy SR, Mistry J, Mitchell AL, Potter SC, Punta M, Qureshi M, Sangrador-Vegas A, Salazar GA, Tate J, Bateman A (2016) The Pfam protein database: towards a more sustainable future. Nucleic Acids Res 44:D279-D285

Gornicki P, Zhu H, Wang J, Challa GS, Zhang Z, Gill BS, Li W (2014) The chloroplast view of the evolution of polyploid wheat. New Phytol 204:704-714

Hayashi N, Inoue H, Kato T, Funao T, Shirota M, Shimizu T, Kanamori H, Yamane H, Hayano-Saito Y, Matsumoto T, Yano M, Takatsuji H (2010) Durable panicle blast-resistance gene $P b l$ encodes an atypical CC-NBS-LRR protein and was generated by acquiring a promoter through local genome duplication. Plant J 64:498-510

Hernandez P, Martis M, Dorado G, Pfeifer M, Gálvez S, Schaaf S, Jouve N, Šimková H, Valárik M, Doležel J, Mayer KFX (2012) Next-generation sequencing and syntenic integration of flowsorted arms of wheat chromosome 4A exposes the chromosome structure and gene content. Plant J 69:377-386 
Huang L, Brooks SA, Li W, Fellers JP, Trick HN, Gill BS (2003) Mapbased cloning of leaf rust resistance gene $\operatorname{Lr} 21$ from the large and polyploid genome of bread wheat. Genetics 164:664-665

Hurni S, Brunner S, Buchmann G, Herren G, Jordan T, Krukowski P, Wicker T, Yahiaoui N, Mago R, Keller B (2013) Rye Pm8 and wheat $P m 3$ are orthologous genes and show evolutionary conservation of resistance function against powdery mildew. Plant J 76:957-969

Islamov B, Peusha H, Jakobson I, Järve K (2015) Triticum militinae introgressions into bread wheat affect host responses to powdery mildew challenge. In: Poster session presented at 14th international cereal rusts and powdery mildews conference, Helsing $\varnothing$, Denmark, 5-8 July 2015

Ivaničová Z, Jakobson I, Reis D, Šafář J, Milec Z, Abrouk M, Doležel J, Järve K, Valárik M (2016) Characterization of new allele influencing flowering time in bread wheat introgressed from Triticum militinae. New Biotechnol 33:718-727

IWGSC (2014) A chromosome-based draft sequence of the hexaploid bread wheat (Triticum aestivum) genome. Science 345:1251788

IWGSC (2018) Shifting the limits in wheat research and breeding through a fully annotated and anchored reference genome sequence. Science 361:eaar7191

Jakobson I, Peusha H, Timofejeva L, Järve K (2006) Adult plant and seedling resistance to powdery mildew in a Triticum aestivum $\times$ Triticum militinae hybrid line. Theor Appl Genet 112:760-769

Jakobson I, Reis D, Tiidema A, Peusha H, Timofejeva L, Valárik M, Kladivová M, Šimková H, Doležel J, Järve K (2012) Fine mapping, phenotypic characterization and validation of non-race-specific resistance to powdery mildew in a wheat-Triticum militinae introgression line. Theor Appl Genet 125:609-623

Järve K, Peusha HO, Tsymbalova J, Tamm S, Devos KM, Enno TM (2000) Chromosome location of a Triticum timopheevi-derived powdery mildew resistance gene transferred to common wheat. Genome 43:377-381

Järve K, Jakobson I, Enno T (2002) Tetraploid wheat species Triticum timopheevi and Triticum militinae in common wheat improvement. Acta Agron Hung 50:463-477

Jia J, Devos KM, Chao S, Miller TE, Reader SM, Gale MD (1996) RFLP-based maps of the homoeologous group- 6 chromosomes of wheat and their application in the tagging of $P m 12$, a powdery mildew resistance gene transferred from Aegilops speltoides to wheat. Theor Appl Genet 92:559-565

King J, Grewal S, Yang C, Hubbart-Edwards S, Scholefield D, Ashling S, Broomfield D, Howells C, King IP (2017) A step change in the transfer of interspecific variation into wheat from its wild relatives. In: Buerstmayr H, Lang-Mladek C, Steiner B, Michel S, Buerstmayr M, Lemmens M, Vollmann J, Grausgruber H (eds) Proceedings of the 13th international wheat genetics symposium, Tulln, Austria, 23-28 April 2017. BOKU-University of Natural Resources and Life Sciences, Vienna, p. 40

Krattinger SG, Lagudah ES, Spielmeyer W, Singh RP, Huerta-Espino J, McFadden H, Bossolini E, Selter LL, Keller B (2009) A putative $\mathrm{ABC}$ transporter confers durable resistance to multiple fungal pathogens in wheat. Science 323:1360-1363

La Camera S, Balagué C, Geoffroy P, Legrand M, Feussner I, Roby D, Heitz T (2009) The Arabidopsis patatin-like protein 2 (PLP2) plays an essential role in cell death execution and differentially affects biosynthesis of oxylipins and resistance to pathogens. Mol Plant Microbe Interaction 22:469-481

Leath S, Bowen KL (1989) Effects of powdery mildew, triadimenol seed treatment and triadimenol foliar sprays on yield of winter wheat in North Carolina. Phytopathology 79:152-155

Leroy P, Guilhot N, Sakai H, Bernard A, Choulet F, Theil S, Reboux S, Amano N, Flutre T, Pelegrin C, Ohyanagi H, Seidel M, Giacomoni F, Reichstadt M, Alaux M, Gicquello E, Legeai F, Cerutti
L, Numa H, Tanaka T, Mayer K, Itoh T, Quesneville H, Feuillet C (2011) TriAnnot: a versatile and high performance pipeline for the automated annotation of plant genomes. Front Plant Sci 3:1-14

Li ZK, Luo LJ, Mei HW, Paterson AH, Zhao XH, Zhong DB, Wang YP, Yu XQ, Zhu L, Tabien R, Stansel JW, Ying CS (1999) A "defeated" rice resistance gene acts as a QTL against a virulent strain of Xanthomonas oryzae pv. oryzae. Mol Gen Genet 261:58-63

Lillemo M, Lu Q (2015) A meta analysis of partial resistance loci to powdery mildew in wheat. In: Paper presented at 14 th international cereal rusts and powdery mildews conference, Helsing ør, Denmark, 5-8 July 2015

Lukaszewski AJ (1995) Physical distribution of translocation breakpoints in homoeologous recombinants induced by the absence of the $P h l$ gene in wheat and triticale. Theor Appl Genet 90:714-719

Lukaszewski AJ (2015) Introgressions between wheat and rye. In: Molnár-Láng M, Ceoloni C, Doležel J (eds) Alien introgression in wheat. Springer, New York, pp 163-190

Luo MC, Yang ZL, Kota RS, Dvořák J (2000) Recombination of chromosome $3 A^{m}$ and $5 A^{m}$ of Triticum monococcum with homeologous chromosomes $3 A$ and $5 A$ of wheat: the distribution of recombination across chromosomes. Genetics 154:1301-1308

Lutz J, Limpert E, Bartos P, Zeller FJ (1992) Identification of powdery mildew resistance genes in common wheat (Triticum aestivum L.). Plant Breed 108:33-39

Mago R, Tabe L, Vautrin S, Šimková H, Kubaláková M, Upadhyaya N, Berges H, Kong X, Breen J, Doležel J, Appels R, Ellis JG, Spielmeyer W (2014) Major haplotype divergence including multiple germin-like protein genes, at the wheat $S r 2$ adult plant stem rust resistance locus. BMC Plant Biol 14:379

Mago R, Vautrin S, Šimková H, Bansal U, Luo MC, Rouse M, Karaoglu H, Periyannan S, Kolmer J, Jin Y, Ayliffe MA, Bariana H, Park RF, McIntosh R, Doležel J, Bergès H, Spielmeyer W, Lagudah ES, Ellis JG, Dodds PN (2015) The wheat Sr50 gene reveals rich diversity at a cereal disease resistance locus. Nat Plants 1:15186

Marchler-Bauer A, Bo Y, Han L, He J, Lanczycki CJ, Lu S, Chitsaz F, Derbyshire MK, Geer RC, Gonzales NR, Gwadz M, Hurwitz DI, Lu F, Marchler GH, Song JS, Thanki N, Wang Z, Yamashita RA, Zhang D, Zheng C, Geer LY, Bryant SH (2017) CDD/SPARCLE: functional classification of proteins via subfamily domain architectures. Nucleic Acids Res 45:D200-D203

Michaels SD, Amasino RM (1999) A robust method for detecting single-nucleotide changes as polymorphic markers by PCR. Plant $\mathrm{J}$ 14:381-385

Michelmore RW, Meyers BC (1998) Clusters of resistance genes in plants evolve by divergent selection and a birth-and-death process. Genome Res 8:1113-1130

Mondal S, Rutkoski JE, Velu G, Singh PK, Crespo-Herrera LA, Guzmán C, Bhavani S, Lan C, He X, Singh RP (2016) Harnessing diversity in wheat to enhance grain yield, climate resilience, disease and insect pest resistance and nutrition through conventional and modern breeding approaches. Front Plant Sci 7:991

Periyannan S, Moore J, Ayliffe M, Bansal U, Wang X, Huang L, Deal K, Luo M, Kong X, Bariana H, Mago R, McIntosh R, Dodds P, Dvorak J, Lagudah E (2013) The gene Sr33, an ortholog of barley Mla genes, encodes resistance to wheat stem rust race Ug99. Science 341:786-788

Rajaraman J, Douchkov D, Hensel G, Stefanoto FL, Gordon A, Ereful N, Caldararu OF, Petrescu AJ, Kumlehn J, Boyd LA, Schweizer P (2016) An LRR/malectin receptor-like kinase mediates resistance to non-adapted and adapted powdery mildew fungi in barley and wheat. Front Plant Sci 7:1836

Riley R, Chapman V (1958) Genetic control of the cytologically diploid behavior of hexaploid wheat. Nature 182:713-715 
Roberts MA, Reader SM, Dalgliesh C, Miller TE, Foote TN, Fish LJ, Snape JW, Moore G (1999) Induction and characterization of Phl wheat mutants. Genetics 153:1909-1918

Röder MS, Korzun V, Wendehake K, Plaschke J, Tixier MH, Leroy P, Ganal MW (1998) A microsatellite map of wheat. Genetics 149:2007-2023

Saintenac C, Zhang W, Salcedo A, Rouse MN, Trick HN, Akhunov E, Dubcovsky J (2013) Identification of wheat gene Sr35 that confers resistance to Ug99 stem rust race group. Science 341:783-786

Sánchez-Martín J, Steuernagel B, Ghosh S, Herren G, Hurni S, Adamski N, Vrána J, Kubaláková M, Krattinger SG, Wicker T, Doležel J, Keller B, Wulff BBH (2016) Rapid gene isolation in barley and wheat by mutant chromosome sequencing. Genome Biol 17:221

Sears ER (1977) Induced mutant with homoeologous pairing in common wheat. Can J Genet Cytol 19:585-593

Sekhwal MK, Li PC, Lam I, Wang XE, Cloutier S, You FM (2015) Disease resistance gene analogs in plants. Int $\mathrm{J}$ Mol Sci 16:19248-19290

Šimková H, Svensson JT, Condamine P, Hřibová E, Suchánková P, Bhat PR, Bartoš J, Šafář J, Close TJ, Doležel J (2008) Coupling amplified DNA from flow-sorted chromosomes to high-density SNP mapping in barley. BMC Genom 9:294

Šimková H, Safář J, Kubaláková M, Suchánková P, Č́íhalíková J, Robert-Quatre H, Azhaguvel P, Weng Y, Peng J, Lapitan NLV, Ma Y, You FM, Luo M, Bartoš J, Doležel J (2011) BAC libraries from wheat chromosome 7D: efficient tool for positional cloning of aphid resistance genes. J Biomed Biotechnol 2011:302543

Steuernagel B, Periyannan SK, Hernández-Pinzón I, Witek K, Rouse MN, Yu G, Hatta A, Ayliffe M, Bariana H, Jones JDG, Lagudah ES, Wulff BBH (2016) Rapid cloning of disease-resistance genes in plants using mutagenesis and sequence capture. Nat Biotechnol 34:652-655

Thind AK, Wicker T, Šimková H, Fossati D, Moullet O, Brabant C, Vrána J, Doležel J, Krattinger S (2017) Rapid cloning of genes in hexaploid wheat using cultivar-specific long-range chromosome assembly. Nat Biotechnol 35:793-796
Tiwari VK, Wang S, Sehgal S, Vrána J, Friebe B, Kubaláková M, Chhuneja P, Doležel J, Akhunov E, Kalia B, Sabir J, Gill BS (2014) SNP discovery for mapping alien introgressions in wheat. BMC Genom 15:273

Tsõmbalova J, Karafiátová M, Vrána J, Kubaláková M, Peusha H, Jakobson I, Järve M, Valárik M, Doležel J, Järve K (2016) A haplotype specific to North European wheat (Triticum aestivum L.). Genet Resour Crop Evol 64:653-664

Untergasser A, Cutcutache I, Koressaar T, Ye J, Faircloth BC, Remm M, Rozen SG (2012) Primer3: new capabilities and interfaces. Nucleic Acids Res 40:e115

Valárik M, Linkiewicz AM, Dubcovsky J (2006) A microcolinearity study at the earliness per se gene Eps-Am 1 region reveals an ancient duplication that preceded the wheat-rice divergence. Theor Appl Genet 112:945-957

Valkoun JJ (2001) Wheat pre-breeding using wild progenitors. Euphytica 119:17-23

van der Biezen EA, Jones JDG (1998) The NB-ARC domain: a novel signalling motif shared by plant resistance gene products and regulators of cell death in animals. Curr Biol 8:R226-R227

Wicker T, Yahiaoui N, Guyot R, Schlagenhauf E, Liu ZD, Dubcovsky J, Keller B (2003) Rapid genome divergence at orthologous low molecular weight glutenin loci of the $\mathrm{A}$ and $\mathrm{A}^{\mathrm{m}}$ genomes of wheat. Plant Cell 15:1186-1197

Winfield MO, Allen AM, Burridge AJ, Barker GLA, Benbow HR, Wilkinson PA, Coghill J, Waterfall C, Davassi A, Scopes G, Pirani A, Webster T, Brew F, Bloor C, King J, West C, Griffiths S, King I, Bentley AR, Edwards KJ (2016) High-density SNP genotyping array for hexaploid wheat and its secondary and tertiary gene pool. Plant Biotechnol J 14:1195-1206

Ye ZM, Ting JPY (2008) NLR, the nucleotide-binding domain leucinerich repeat containing gene family. Curr Opin Immunol 20:3-9

Zamir D (2001) Improving plant breeding with exotic genetic libraries. Nat Rev Genet 2:983-989 